\title{
Rodrigo Gil de Hontañón y la Colegiata de Peñaranda de Duero (Burgos): aportación a su estudio
}

\author{
Juan Escorial Esgueva \\ Universidad de Salamanca \\ juanescorial@usal.es
}

Francisco de Zúñiga y Avellaneda y María Enríquez de Cárdenas, III condes de Miranda, desarrollaron, a lo largo del primer tercio del siglo XVI, una intensa actividad promotora en la villa burgalesa de Peñaranda de Duero. De ello, dan buena muestra las diferentes construcciones que modificaron de forma sustancial la fisonomía del caserío medieval, como su magnífica residencia palaciega (Lampérez, 1912: 146-151; Carazo, 1997: 505-544), el convento de Nuestra Señora de la Concepción y el hospital de la Piedad (Zaparaín, 2013: 280-289; Escorial, 2019: 109-114). Todas estas actuaciones dotaron a la villa de un singular protagonismo dentro del dominio territorial de los Zúñiga, convirtiéndose en el núcleo principal de sus estados.

A ellas se sumarían diversas intervenciones que tuvieron como objeto la transformación del cercano monasterio premonstratense de Santa María de La Vid, entre las que destaca la construcción de la capilla mayor de su iglesia. Esta obra, que sigue la fisonomía de las construcciones promovidas por el linaje de los Velasco en el ámbito burgalés durante las décadas iniciales del quinientos, fue costeada por el III conde de Miranda y su hermano, el cardenal Íñigo López de Mendoza (Alonso, 2003b: 279-295; 2003a: 45-57; Escorial, 2019: 114122). Se trataba, a su vez, del espacio elegido por los III condes para situar sus enterramientos, según recogieron en el testamento que el matrimonio redactó de forma conjunta en Torrijos, el 10 de enero de 1533, al afirmar que era el ámbito en el que estaban «nuestros antecesores enterrados y do hemos de estar nosotros» (Escorial, 2019: 116).

Sin embargo, tras el fallecimiento del cardenal, en 1535, y del III conde, en 1536¹, su viuda abandonó la idea de ocupar la tumba concebida en la capilla de La Vid para emprender, en Peñaranda de Duero, un ambicioso proyecto personal en el que situaría su nuevo enterramiento. Se trataba, pues, de la construcción de un gran templo que ven- dría a sustituir a las antiguas parroquias de San Miguel y San Martín, situadas en la ladera del castillo, «apartadas de la conversación de la gente y vecindad» y cuyos caracteres arquitectónicos estarían lejos de las expectativas puestas por la familia en la renovación de la villa. Por ello, a finales de 1539, la condesa viuda solicitó permiso al obispo de Osma, Pedro Álvarez de Acosta, para levantar el nuevo edificio² (Zaparaín, 2013: 287; Escorial, 2016: 97).

Aunque las características de este primer proyecto son completamente desconocidas, parece que la condesa siguió personalmente el desarrollo de las obras, como refleja el hecho de que en el testamento que otorgó el 26 de enero de 1544 ordenara que el templo "se haga y acabe [...] conforme a la traça que lleba y está dada por los canteros y oficiales que la hacen, la qual está en mi cámara»³. A su vez, expresaba el deseo de que «por quanto yo hago en esta mi villa de Peñaranda una yglessia de la vocación de señora Santa Ana en serviçio de nuestro señor», su tumba debería situarse «en lo prinçipal de la [...] capilla mayor» de dicho templo 4 .

No obstante, consciente de las dificultades de la construcción y de la posibilidad de que las obras se dilataran en el tiempo, declararía también que «si por ventura al tiempo de mi fallecimiento la dicha capilla mayor [... ] no estubiere acabada» mandaba que su cuerpo fuera sepultado temporalmente en otra de las fundaciones protegidas por la familia ${ }^{5}$. En su codicilo, fechado el 22 de marzo de 1544, ratifica nuevamente esta decisión, pero hace algunas matizaciones, al rechazar que su cuerpo se enterrara en otro lugar, mandando que fuera "depositado en la sacristía» del nuevo templo, a la espera de que se finalizaran las obras de la capilla mayor 6 .

María Enríquez de Cárdenas falleció el 14 de mayo de $1544^{7}$, siendo, a partir de ese momento, sus hijos, Gaspar de Zúñiga y Pedro Núñez de Avellaneda, los encargados de 


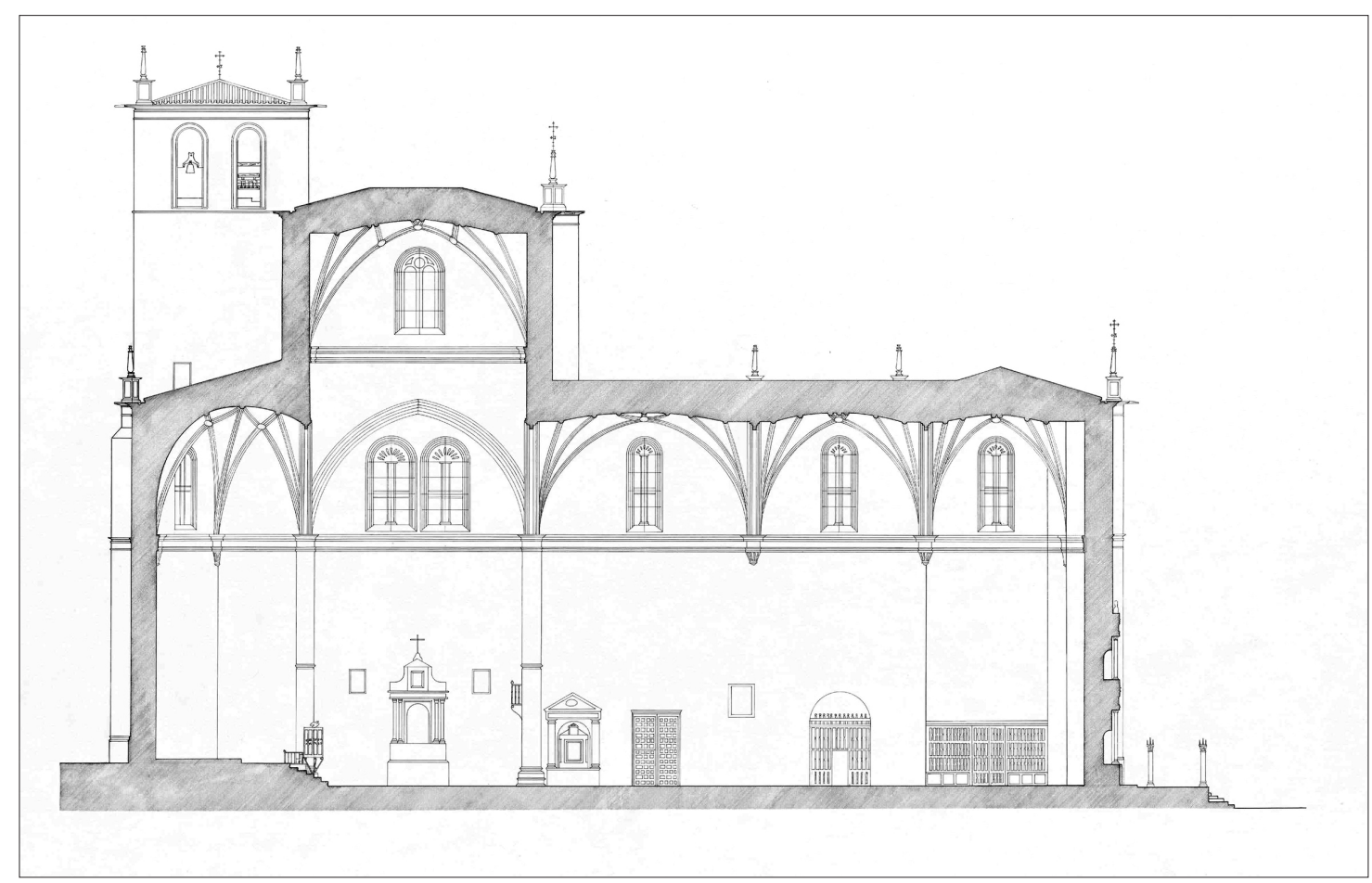

1. Sección longitudinal de la colegiata de Peñaranda de Duero (Burgos). Jesús Peñalba Aguilera

la materialización del proyecto, siguiendo las últimas voluntades de la condesa. Estos atendieron con diligencia todo lo relacionado con el avance de las obras, si bien delegaron parte de sus responsabilidades en diferentes mayordomos que se ocuparían de «entender en la fábrica y obra que en ella se haçe» ${ }^{8}$.

Núñez de Avellaneda nombró, a finales de 1544, al maestro de cantería Pedro de Landa como aparejador del templo, obligándose a «obrar y azer la dicha obra [...] conforme a una traça y condiciones» que el hijo de la condesa le había entregado, con el consentimiento y la aprobación de Bartolomé de Pierredonda ${ }^{9}$.

Al año siguiente, el 18 de octubre de 1545, Pierredonda fue nombrado «visitador y tasador de la dicha hobra», y, en este preciso momento, declara que es necesario «mudar en el cuerpo de la dicha yglesia quatro estribos más debajo de lo que estaban [...] por ensanchar y dar espaçio a la capilla que está junto al cruzzero ${ }^{10}$. Estas modificaciones, que llevaría a cabo Landa de forma inmediata ${ }^{11}$, revelan los problemas que el diseño original tuvo desde sus primeros momentos. Poco después, este maestro se encargaría de realizar la cimentación del resto del edificio ${ }^{12} \mathrm{e}$, incluso, colaboró en el diseño de alguno de sus elementos, pues consta que hizo «dos trazas», aunque se desconoce para qué estaban destinadas ${ }^{13}$.

Pese a estos cambios, no parece que el desarrollo de la construcción agradara a sus responsables, que no tardarían en plantear una importante transformación de su diseño. En este contexto debe situarse la presencia, el 20 de abril de 1549, de Pedro de Rasines, que fue requerido para «ver y tasar la obra de la dicha yglesia» ${ }^{14}$ (Alonso, 2003b: 305306) que, en ese momento, apenas se habían concluido los muros de los brazos del crucero ${ }^{15} \mathrm{y}$, parece ser, que el resultado no era del agrado de los promotores. Por ello, unos meses después, se encomienda a Landa la continuación de las obras «conforme a la traza y instruçión hecha por maestro de cantería que le diere» Gaspar de Zúñiga ${ }^{16}$.

En esos momentos don Gaspar ocupaba la cátedra de Teología en la Universidad de Salamanca. Este hecho parece explicar la circunstancia de que el maestro elegido para ejecutar el diseño definitivo del templo fuera Rodrigo Gil de Hontañón ${ }^{17}$ que, de forma coetánea se estaba encargando 


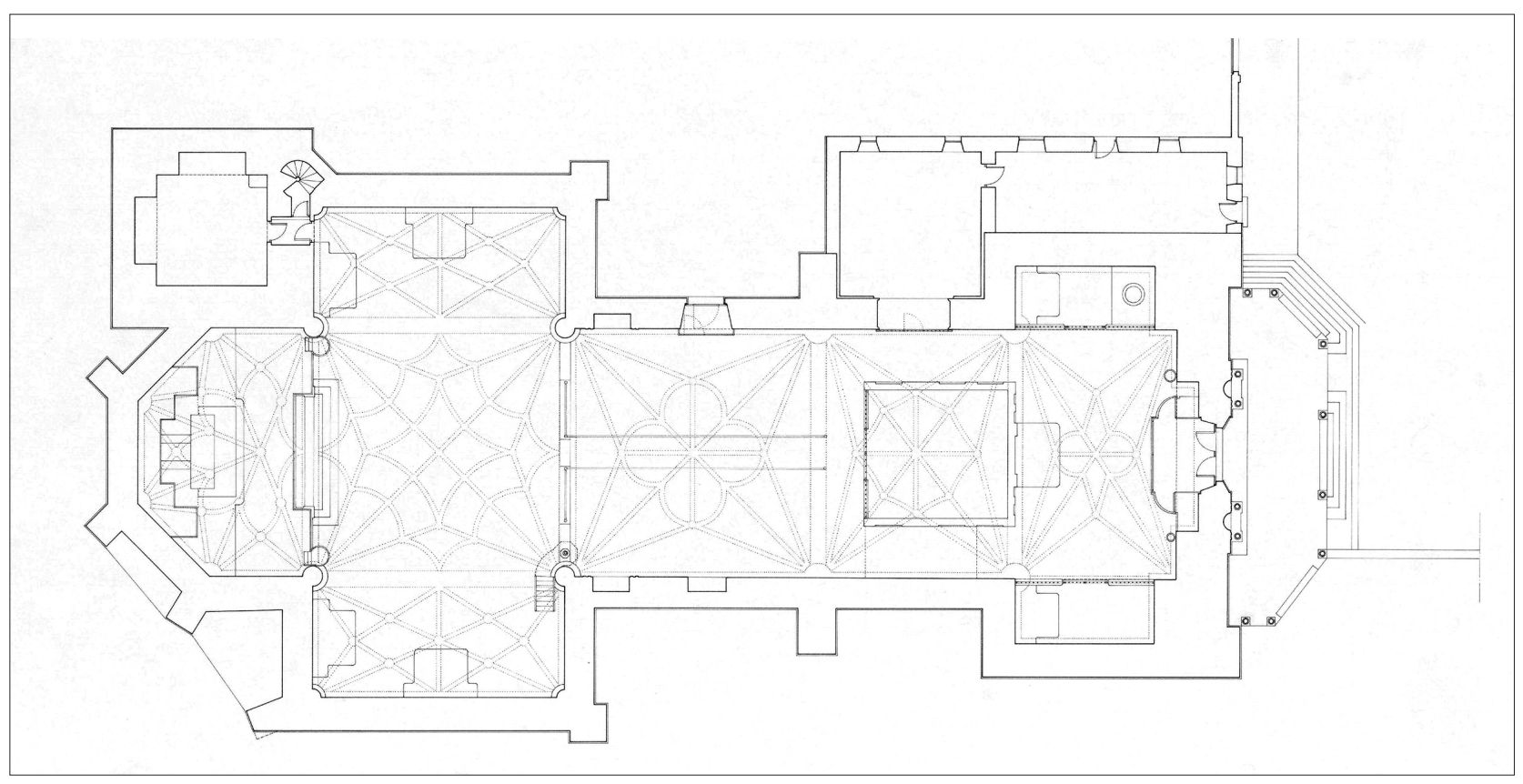

2. Planta de la Colegiata de Peñaranda de Duero (Burgos). Jesús Peñalba Aguilera

de los proyectos más importantes de la ciudad del Tormes (Hoag, 1985; Casaseca, 1988). Al parecer, este maestro entregó a don Gaspar una planta del templo a la que se alude frecuentemente en la documentación y en la que figuraba la firma del hijo de la III condesa ${ }^{18}$, así como «otro papel en que estaban debujados los moldes para la dicha obra», en los que se incluían los perfiles de las diferentes molduras que debían emplearse en el edificio ${ }^{19}$ y que facilitarían el trabajo a los responsables de las obras.

La propuesta de Rodrigo Gil de Hontañón se vinculaba a las obras que, en esos mismos años estaba llevando en la iglesia del convento de San Esteban y en la ampliación de la capilla del colegio del arzobispo Fonseca (Hoag, 1985: 137-142 y 170-178; Casaseca, 1988: 179-191). Pese a las diferencias de escala entre estos proyectos con la obra burgalesa, todos ellos coinciden en la configuración de espacios de carácter funerario estructurados a través de una planta de cruz latina con brazos de escaso desarrollo y un potente cimborrio sobre el crucero, de planta cuadrangular y cubierto por una compleja bóveda de crucería (Gómez, 1998: 73, 75; Ibáñez y Alonso, 2017: 194-199) [1] y [2].

El nuevo proyecto se puso en práctica de forma inmediata, pues consta que Pedro de Landa se dispuso a «abrir los cimientos de la delantera de la dicha yglesia [...] y de las capillas últimas junto a ella hasta los agujeros donde se a de elexir el coro» ${ }^{20}$, obligándose a acabar «la torre y coro y sacristía» ${ }^{21}$ según este mismo diseño. Sin embargo, parece ser que aprovecharon algunos elementos ya realizados, como el cuerpo inferior de la torre, como han puesto de manifiesto diversos estudios (Sánchez, 2011: 147; Sánchez et al., 2014: 97-108).

No obstante, las dificultades para adaptar lo construido hasta entonces con el nuevo planteamiento exigieron que el arquitecto visitara la villa burgalesa en 1551. En esta visita procedió a tasar los materiales existentes al pie de la obra y dictaminó las distintas medidas que debían seguirse para que la construcción pudiera continuar su desarrollo, ordenando el derribo de «los estribos de la capilla prinçipal»"22. Landa sería, de nuevo, el encargado de continuar las obras en los años siguientes, comprometiéndose a realizar las «vóbedas para çerrar las capilllas e crucero y cuerpo de la dicha yglesia»23, por lo que el templo ya debía alcanzar una altura considerable, aunque su intervención modificó algunos de los elementos definidos por Gil de Hontañón, especialmente en el arranque de la nave, que no se hizo «conforme a la traça» ${ }^{24}$. 


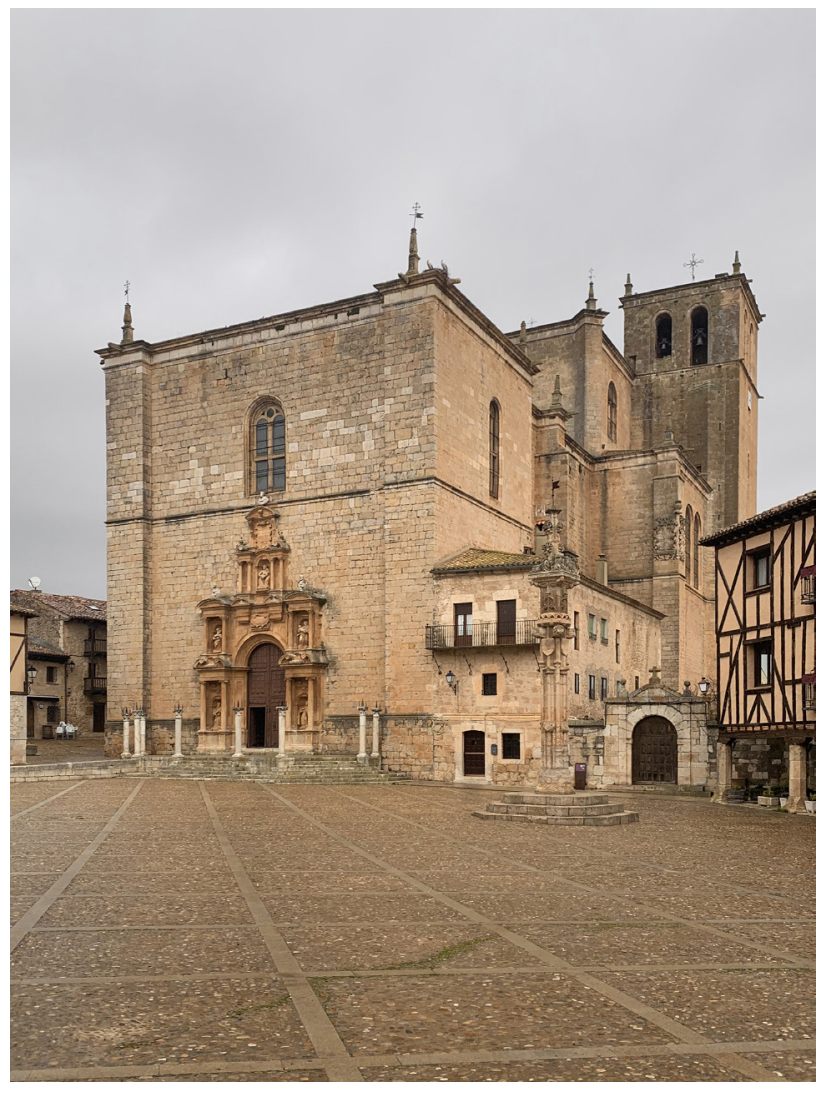

3. Colegiata de Peñaranda de Duero (Burgos) (fotografía del autor)

Tras el fallecimiento de Landa, en $1562^{25}$, la dirección de las obras pasó a Pedro Díez de Palacios (Losada, 2004: 375-402; 2007: 105), que se obligaría con Pedro Núñez de Avellaneda a «subir e zerrar el cuerpo e cimborrio e capillas hornezinas e torre de la dicha yglesia, muy bien acabada conforme a la traza e como estaba comenzada y echa la planta della $»^{26}$. Sin embargo, en 1569 abandonaría la obra "por yrse a Sebilla, donde se quedó por maestro» de la catedral hispalense, lo que generaría un complejo pleito en la Chancillería vallisoletana en el que se le reclamarían las responsabilidades derivadas de dicho abandono. Poco después, los maestros Juan López de Obieta y Pedro de Rasines visitarían el templo para dar su parecer sobre las obras ${ }^{27}$, encargándose, este último, de la dirección de las mismas hasta su fallecimiento, en 1572 (Río, 2000: 333-335).

Los diferentes problemas derivados del desinterés de los descendientes de la III condesa, así como los distintos

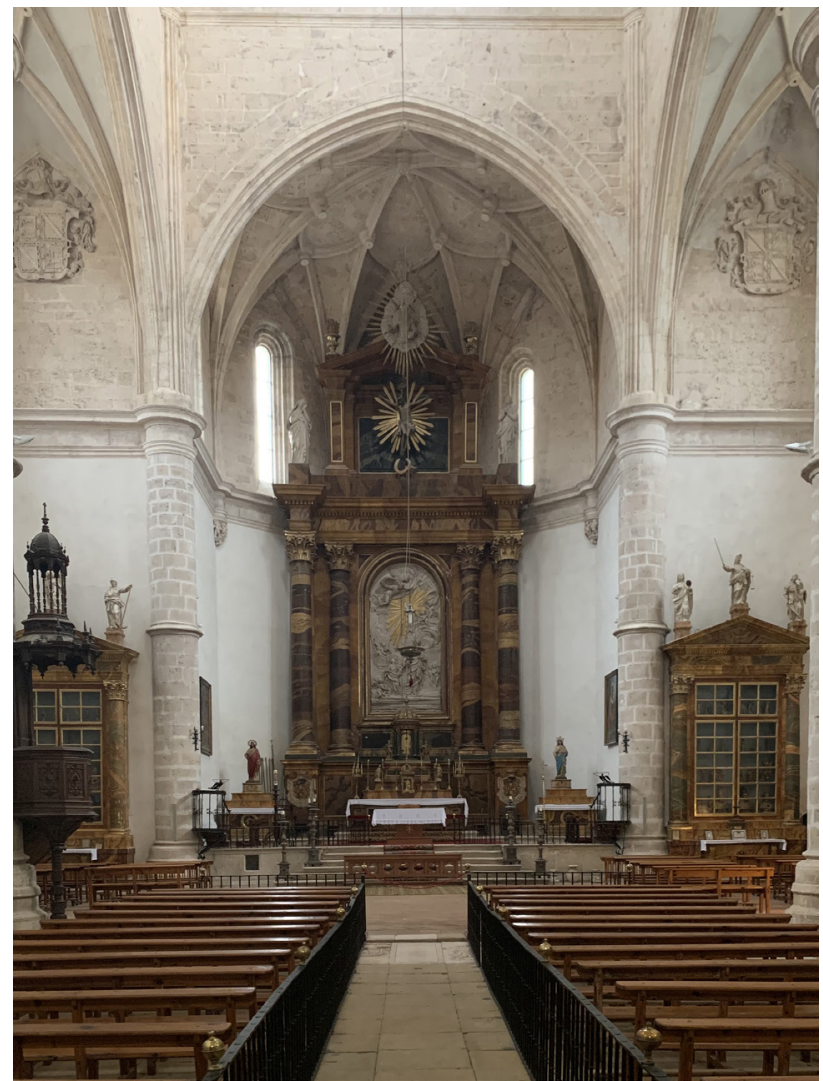

4. Interior de la colegiata de Peñaranda de Duero (Burgos) (fotografía del autor)

cambios de maestro encargado de la dirección de las obras, ralentizaron la construcción del edificio e, incluso, alteraron algunos de los elementos definitorios del proyecto de Rodrigo Gil de Hontañón. De hecho, se eliminaron «unos mármoles inclusos en la mesma pared" que soportarían los arcos de la capilla mayor, recogidos en la traza ${ }^{28}$ y que no fueron ejecutados, así como otras modificaciones en el tipo de cornisas escogidas o el número de vanos del cimborrio, que se redujeron a la mitad ${ }^{29}$. A finales de la centuria, los VI condes de Miranda, Juan de Zúñiga y Avellaneda y María de Zúñiga completaron la realización del cimborio ${ }^{30}$. No obstante, su conclusión no se llevaría a cabo hasta avanzado el setecientos, cuando Antonio López de Zúñiga, XIII conde de Miranda, retomaría los proyectos de sus antepasados, manteniendo los elementos definitorios del proyecto original de Rodrigo Gil de Hontañón (Zaparaín, 1993: 581-588; 2002: 379-381; Zaparaín y Escorial, 2019: 138-139) [3] y [4]. 


\section{Notas}

1 Archivo General de Simancas, Valladolid (AGS, Valladolid), Estado, leg. 38, ff. 11, 33, 65, 286; Archivo Diocesano de Burgos, Burgos (ADBu, Burgos), Peñaranda de Duero, leg. 10, Bullas y papeles originales con los autos que tocan a la jurisdicción desta collegial de Peñaranda, f. 2v.

2 Pese a que los antiguos templos continuaron en funcionamiento, como había mandado el obispo de Osma, pasaron a un segundo plano, y muchos de los enterramientos fueron trasladados a la nueva iglesia desde fechas tempranas. Sobre ello: Archivo Histórico Provincial de Burgos, Burgos (AHPBu, Burgos). Prots. 5250/3, f. 97; 5251, f. 281.

3 ADBu, Burgos, Peñaranda de Duero, leg. 10, Bullas y papeles originales..., doc. cit., ff. 10-10v.

4 Ibidem, ff. $2 \mathrm{v}-3$.

5 Ibid., ff. $2 \mathrm{v}-3$.

6 Ibid., f. 41v.

7 Archivo Histórico de la Nobleza, Toledo (AHNob, Toledo), Baena, c. 71, d. 253.

8 AHPBu, Burgos, Prots. 5259/2, ff. 28-29v; 5259/6, ff. 52-53v; 5259/9, ff. 94-94v.

9 ADBu, Burgos, Peñaranda de Duero, leg. 6, Obligación de Pedro de Landa y sus fiadores, 22/12/1544.

10 Aunque se ha considerado que Pierredonda fue quien realizó el primero de los proyectos, como han mantenido diversos investigadores desde el estudio de Ibáñez (1989: 399), los datos documentales apuntan a que únicamente visitó y tasó las obras (Cadiñanos, 1993: 119; Zaparaín, 2013: 287).

11 ADBu, Burgos, Peñaranda de Duero, leg. 6, Cuentas, 04/12/1545.

12 Ibid., Cuentas, 25/05/1547.

13 Ibid., Cuentas, 09/05/1549.

$14 \mathrm{Ibid}$., Cuentas, 08/05/1549, 13/09/1549.

15 Ibid., Cuentas, 09/05/1549.

$16 \mathrm{Ibid}$., Condiciones para la obra de la iglesia, 13/10/1549.

17 Casaseca (1988: 319) sospechó la posibilidad de que Rodrigo Gil de Hontañón realizara el proyecto de Peñaranda, lo cual fue confirmado documentalmente por lbáñez (1989: 398-401).

18 Archivo de la Real Chancillería de Valladolid, Valladolid (ARChV, Valladolid), Pleitos Civiles, Zarandona y Walls, Fenecidos, caja 177, 2, 16/12/1563, visita de Juan González de Pontones y Pedro Díez de Palacios a la obra de Peñaranda de Duero; 13/11/1570, f. 4

19 Ibid.

20 ADBu, Burgos, Peñaranda de Duero, leg. 6, Cuentas, 13/09/1549.

$21 \mathrm{lbid}$., Condiciones para la obra de la iglesia, 13/10/1549.

22 Ibid., Cuentas, 15/07/1551. Citado por Ibáñez (1989: 400).

23 Ibid., Cuentas, 08/05/1550; 15/07/1551; 21/09/1552; 14/03/1554. AHPBu, Burgos, Prot. 5250/3, ff. 216-221V.

24 ARChV, Valladolid, Pleitos Civiles, Zarandona y Walls, Fenecidos, caja 177, 2, 16/12/1563.

25 Había otorgado testamento el 13 de febrero de 1562. AHPBu, Burgos, Prot. 5253, ff. 93v-95.

26 ADBu, Burgos, Peñaranda de Duero, leg. 6, 17/02/1564, 23/02/1564, ff. 1v-2. Sobre las intervenciones de esta época: ibid. Contrato con Juan de Arana, cantero, por sacar piedra, 09/03/1565; Memoria de los materiales que son menester si Dios Nuestro Señor fuere servido para este año de mil y quinientos y sesenta y siete; Cartas de obligación de Pedro de Zumárraga, 1568, 1569; Carta de obligación de Pedro de la Portilla, cantero, 1569. AHPBu, Burgos, Prots. 5252, f. 71v; 172-173; 10718/3, ff. 200-201.

27 ARChV, Valladolid, Pleitos Civiles, Zarandona y Walls, Fenecidos, caja 177, 2, 13/11/1570, f. 3; 03/07/1571, ff. 27v-28, 32-34v. Sobre su participación en la obra: ADBu, Burgos, Peñaranda de Duero, leg. 6, Cuentas, 1566-1572; Pago a Pedro de Rasines, maestro de cantería, 11/07/1571. AHPBu, Burgos, Prot. 5254, ff. 421-424v.

28 ADBu, Burgos, Peñaranda de Duero, leg. 6. Instrucciones para Diego Núñez, 1592. Recogido por Cadiñanos (1993: 118-124)

29 ARChV, Valladolid, Pleitos Civiles, Zarandona y Walls, Fenecidos, caja 177, 2, 16/12/1563.

30 ADBu, Burgos, Peñaranda de Duero, leg. 6. Memoria del gasto que tiene el cimborrio, 1590; Contrato con Domingo de Dueñas y Ana de Coxeces, 08/02/1591. Carta de pago por el hierro de la cruz del cimborrio, etc.

\section{Bibliografía}

ALONSO RUIZ, Begoña (2003a), «De la capilla gótica a la renacentista. Juan Gil de Hontañón y Diego de Siloé en La Vid», Anuario del Departamento de Historia y Teoría del Arte, n. ${ }^{\circ} 15$, pp. 45-57.

- (2003b), Arquitectura tardogótica en Castilla: los Rasines, Universidad de Cantabria, Santander.

CADIÑANOS BARDECI, Inocencio (1993), «Peñaranda de Duero: notas de historia y arte», Biblioteca. Estudio e investigación, n. 8 , pp. 111-132.

CARAZO LEFORT, Eduardo (1997), «El palacio de los condes de Miranda en Peñaranda de Duero», Academia, n. ${ }^{\circ}$ 85, pp. 505-544. 
CASASECA CASASECA, Antonio (1988), Rodrigo Gil de Hontañón (Rascafría 1500-Segovia 1570), Junta de Castilla y León, Valladolid.

ESCORIAL ESGUEVA, Juan (2016), «La Ribera burgalesa durante el episcopado de Pedro Álvarez de Acosta (1539-1563): entre el ornato del culto y la perdurabilidad de la memoria», Biblioteca. Estudio e investigación, n. ${ }^{\circ}$ 31, pp. 91-121.

- (2019), "Los III condes de Miranda y sus fundaciones religiosas: entre el recuerdo familiar y la exaltación del linaje», Ars Longa, n. 28, pp. 109-122.

GÓMEZ MARTínEZ, Javier (1998), El gótico español de la Edad Moderna: bóvedas de crucería, Universidad de Valladolid, Valladolid.

HOAG, John D. (1985), Rodrigo Gil de Hontañón. Gótico y renacimiento en la arquitectura española del siglo XVI, Xarait, Madrid.

IBÁÑEZ FERNÁNDEZ, Javier y ALONSO RUIZ, Begoña (2016), «El cimborrio en la arquitectura española de la Edad Media a la Edad Moderna. Diseño y construcción», Artigrama, n. ${ }^{\circ} 31$, pp. 115-202.

IBÁÑEZ PÉREZ, Alberto C. (1989), «Rodrigo Gil de Hontañón y la iglesia colegial de Peñaranda de Duero (Burgos)», Boletín del Seminario de Estudios de Arte y Arqueología, n. ${ }^{\circ}$ 55, pp. 398-401.

LAMPÉREZ Y ROMEA, Vicente (1912), «El palacio de los condes de Miranda en Peñaranda de Duero (Burgos)», Boletín de la Sociedad Española de Excursiones, vol. XX, n. ${ }^{\circ}$ 2, pp. 146-151.

LOSADA VAREA, Celestina (2004), «Pedro Díez de Palacios y la portada de la iglesia de Gumiel de Izán», Biblioteca. Estudio e investigación, n. ${ }^{\circ} 19$, pp. 377-402.

- (2007), La arquitectura en el otoño del Renacimiento: Juan de Naveda, 1509-1638, Universidad de Cantabria, Santander.

RíO DE LA HOZ, Isabel del (2000), El escultor Felipe Bigarny (h. 1470-1542), Junta de Castilla y León, Valladolid.

SÁNCHEZ RIVERA, José Ignacio (2011), «Las torres del s. XVI en la Ribera del Duero: de la atalaya al mundo urbano». Biblioteca. Estudio e investigación, n. ${ }^{\circ} 26, \mathrm{pp} .137-160$.

SÁNCHEZ RIVERA, José Ignacio, SAN JOSÉ ALONSO, Jesús Ignacio y FERNÁNDEZ MARTíN, Juan José (2014), Ocho torres. Análisis sobre la evolución de campanarios del XVI en la provincia de Burgos, Universidad de Valladolid, Valladolid.

ZAPARAÍN YÁÑEZ, María José (1992), «El patronato del conde de Miranda en la iglesia colegial de Peñaranda de Duero, 1728-1732», en Patronos, promotores, mecenas y clientes. VII Congreso Nacional de Historia del Arte, Universidad de Murcia, Murcia, pp. $581-588$.

- (2002), Desarrollo artístico de la comarca arandina. Siglos XVII y XVIII, Diputación Provincial de Burgos, Burgos.

- (2013), «Con otros ojos. La promoción nobiliar femenina en la Ribera burgalesa del Duero. Siglos XVI y XVIl», Biblioteca. Estudio e investigación, 28, pp. 261-298.

ZAPARAÍN YÁÑEZ, María José y ESCORIAL ESGUEVA, Juan (2019), «Gusto y promoción en el contexto cortesano: los condes de Miranda en el tránsito a la Contemporaneidad», De Arte, 18, pp. 135-155. 